\title{
Medication Errors in a Private Hospital of Bangladesh
}

\author{
Tripti Rani Paul, Md. Ajijur Rahman, Mohitosh Biswas, Mamunur Rashid \\ and Md. Anwar Ul Islam
}

Department of Pharmacy, University of Rajshahi, Rajshahi-6205, Bangladesh

\begin{abstract}
The present study was designed to assess the incidence of various prescription errors with handwritten prescription order for hospitalized patients. Investigation was made in a private hospital situated at the outskirts of Bogra, Bangladesh. A total of 200 prescription orders for the admitted patients in medicine, surgery, orthopedic and diabetes ward of the hospital were evaluated. Prescribing errors were determined by consulting with the standard treatment guidelines, text books and authentic online sources. About 692 (3.46 per prescription) medication related problems (MRPs) were identified from 1234 prescribed drugs. The names of 63 prescribed drugs were not clear due to ambiguous hand writing, the dose strength were found missing for 219 drugs and surprisingly among them 42 drugs were available in the market in multiple dose strengths. More importantly, a total of 366 drug interactions were also identified of which $12.57 \%$ was serious, $53.28 \%$ was significant and $34.15 \%$ was minor. About $15 \%$ prescriptions orders were identified for the patients with kidney and urinary problems and there were no dose adjustment. In $3 \%$ of prescriptions, two drugs having same pharmacological action were prescribed. Although in medical practice single drug prescription is encouraged, the average number of prescribed drugs per prescription was 6.17. These serious prescription errors are the major cause of patient morbidity and mortality, increased length of hospital stay and substantial extra treatment costs.
\end{abstract}

Key words: Prescription errors, Medication related problems (MRPs), Multiple strengths, Single drug prescription, Complex regimen.

\section{Introduction}

Prescribing errors are common in hospital practice internationally. Errors occurred in $1.5 \%$ of medication orders prescribed during the hospital stay in the United Kingdom (UK) (Dean et al., 2002) and in up to 6.2\% of medication orders in the United States of America (USA) (Lesar et al., 1997; Bobb et al., 2004). Such studies (Dean et al., 2002; Lesar et al., 1997; Bobb et al., 2004; Blum et al., 1988; Folli et al., 1987) have used pharmacists to collect data on errors they identified in the course of routine work. Pharmacists in most UK and US hospitals are responsible for medicines management during admission. Identifying and, more importantly, addressing prescribing errors is central to that role (Hawkey et al., 1990).

Various regulatory systems govern the process of prescription generation and dispensing to maximize the safety and efficiency of the product supplied. Community pharmacists have an important role in checking prescription to ensure they are appropriate to dispense. Error can happen in all stages of care process from diagnosis to drug administration. Error is defined as the failure of a planned action to be completed as intended or the use of a wrong plan to achieve an aim (Kohn et al., 1999). Medication errors have been found in a wide variety of clinical environments, ranging from ophthalmology clinics (Mandal et al., 2005) to pediatric critical care units (Rinke et al., 2008). Such errors may lead to prolonged hospitalization, unnecessary diagnostic tests, treatments and deaths (Kozer et al., 2006; Kozer et al., 2005). Error occur as result of two kinds of failure; either the correct action does not proceed as intended (an error of execution) or the original intended action is not correct (an error of planning) (Reason 1990). Writing a prescription is a vital part of a patient's management and studies in New York, Colorado, Utah and Australia have shown rates of adverse events caused by medical mismanagement of $3.7-10.6 \%$ of all admissions (Bates

Correspondence to: Md. Anwar Ul Islam, E-mail: profanwarulislam@yahoo.com 
et al., 1995; Smith 1999). In addition to the human cost, there is an annual financial cost of $£ 500$ million. MEs are more common because of polypharmacy. The majority of errors are not due to reckless behavior on the part of health care providers, but occur as a result of the speed and complexity of the medication use cycle, combined with faulty systems, processes, and conditions that lead people to make mistakes or fail to prevent them (Moyen et al., 2008; Barker et al., 2002).

A clinically meaningful prescribing error occurs when, as a result of a prescribing decision or prescriptionwriting process, there is an unintentional, significant reduction in the probability of treatment being timely and effective, or increase in the risk of harm when compared with generally accepted practice (Dean et al., 2000).

Complex regimen: Prescription of more than three drugs to one patient at the same time.

Wrong combination: implies drug interactions and therapeutic duplication.

The major consequences of medication errors are patient morbidity and mortality (Moyen et al., 2008; Fahimi et al., 2007; Williams, 2007). MEs can affect patients, families and healthcare providers indirectly because of cost implications, prolonged hospital stays and psychological impact, since errors erode public confidence in the health care service (Moyen et al., 2008; Barker et al., 2002; Fahimi et al., 2007; Williams 2007). Previous studies highlighted the problems and identified the causes such as poorly structured prescription on applying hand written technique (Bates et al., 1995; Dean et al., 2000a; Dean et al., 2000b). The prevalence, incidence and nature of prescribing errors for admitted patients were reviewed (Dean et al., 2000). The causes and factors associated with these errors were described (Lewis et al., 2009). The objective of this study was to detect the incidence of prescribing errors for admitted patients, classify and to evaluate the clinical significance of such errors.

\section{Methods}

Study area: A cross-sectional study was conducted in a 450 bed teaching hospital named Rafatullah and TMSS Community Hospital, Bogra, Bangladesh. Data was collected one week in a month during winter season (October to December 2011). Briefly, this involved prescribers hand written in patient medication orders for medical wards only.

Study subjects: A total of 200 patients were selected from Medicine, Surgery, orthopedic and Diabetes wards of the hospital after 24 hours of admission and the patients who are under the control of responsible physician. All the data for the analysis extracted from the patient's case note treatment chart and by interviewing patients using a data collection format.

Data collection process: The prescriptions were considered complete where all information from patient about health and medicine related was present. Data were collected using a structured format to obtain information from patient's cards and medication charts. The content includes demographic variables, date, name of medication, dosages forms, doses, and frequency. Prescribing errors were determined by comparing prescribed drugs with national standard treatment guidelines, textbooks, handbooks and software. Data were edited, coded and entered into SPSS (Statistical package for social service) version 15. Frequency and descriptive statistics were computed to determine specific errors and the overall prevalence of prescribing error. Before the start of data collection, permission was obtained from the hospital management and written consent was obtained from respondents. Moreover, the name of patients was replaced with codes to avoid individual identifiers.

\section{Results and Discussion}

A wide range of different type errors associated with handwritten prescription orders were demonstrated in the present study. These orders were given by the treating physicians for critically admitted patients having one medical problem or more where some of these errors were potentially serious and might cause harm to patients. Such as delay in patient therapy progress, complicated patient medical problem and therapeutic approach that might lead to patient death. Besides this and in terms of prescribing process, most of the errors concerned the selection of the drug strength, ambiguous hand writing and drug- drug interaction.

A total of 200 in patient prescriptions out of 1234 written medication orders during the study period were intervened. These corrected errors were categorized as medication prescribing errors. Clinically meaningful 
prescribing errors occur as a result of prescribing decision and prescription writing process.

Characteristics of interventions and participants: The majority of the patients were male $110(55 \%)$ and female $90(45 \%)$ with $78.5 \%$ of them aged between $18-60$ years. Among these patient $37.5 \%$ and $39 \%$ were uneducated and from primary education level respectively. 67 (33.5\%) had previous hospitalization record and 42 of them were admitted for the same reason (Table 1). This study identified $4.5 \%$ prescription with no admission date of patients. About 188 (94\%) patients received complex regimen.

Table 1. Characteristics of patients admitted to the hospital.

\begin{tabular}{lll}
\hline & Characteristics & Frequency (\%) \\
\hline Age & $<-17$ years & $13(6.5 \%)$ \\
& $18-60$ years & $157(78.5 \%)$ \\
Sex & $>60$ years & $30(15 \%)$ \\
Education level & Male & $110(55 \%)$ \\
& Female & $90(45 \%)$ \\
& Uneducated & $75(37.5 \%)$ \\
Length of stay & Primary & $78(39 \%)$ \\
& Secondary & $31(15.5 \%)$ \\
& $1-5$ days & $162(81 \%)$ \\
Regimen taken & 6-20 days & $29(14.5 \%)$ \\
& Date isn't written & $9(4.5 \%)$ \\
Dose adjustment & Complex & $188(94 \%)$ \\
& Not complex & $12(6 \%)$ \\
& Dose adjustment of patients with renal and & 0 \\
\hline
\end{tabular}

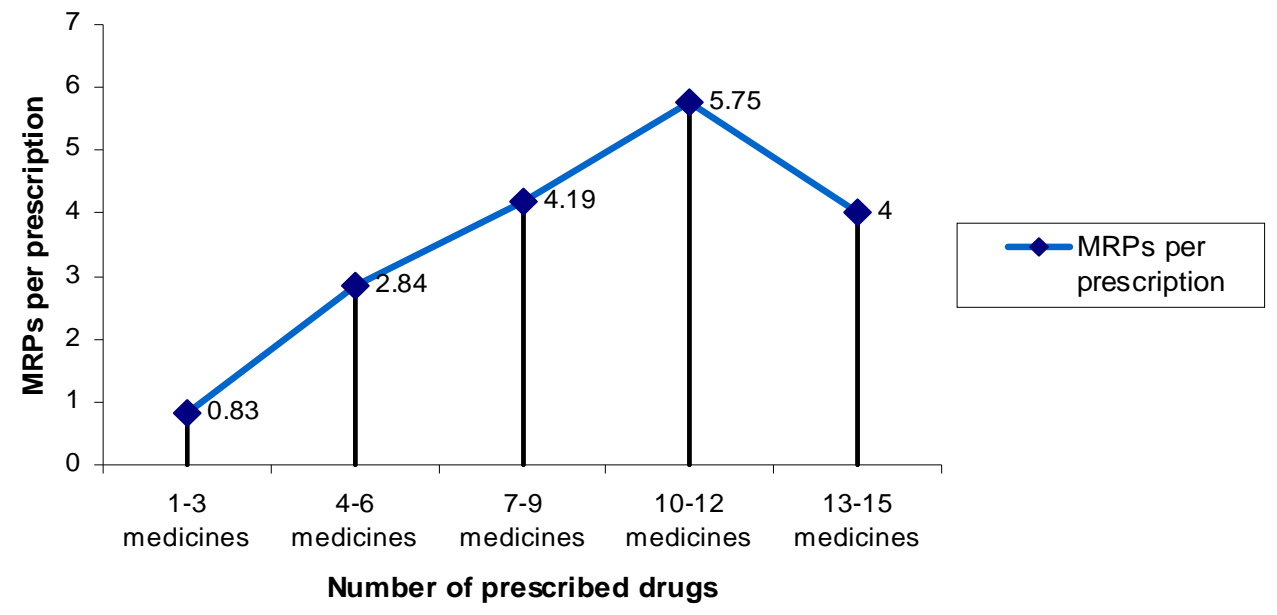

Figure 1. Frequency of medication prescribing errors.

According to the study, the frequency of medication prescribing errors was 692 (3.46 per patient) (Figure 1). Approximately, 113 (7.1\%) prescribing errors were detected out of 1580 medication orders (Al-Dhawailie 2011). This result is lower as compared to our present study.
Medication errors detection and pharmacist intervention were very important factors for quality of pharmacy services provided to needy patients (Bates et al., 1995). Three hundred and twenty-seven MRPs were identified with 308 patients reviewed. The incidence of MRPs was found to be $1.06 \pm 0.85$ per patient reviewed (Castelino et al., 2011). 
As shown in the present study, the most common medication related problems (MRPs) out of 1234 prescribed medicines were with the name of 63 prescribed drugs were not clear due to ambiguous hand writing, strength of medicine were not mentioned in case of 219 prescribed drugs, strength of medicine was not stated for drugs marketed in various strength was 42 (Figure 2)

It might cause serious problem when a right medicine isn't selected due to ambiguous hand writing. On the other hand, when strength of medicine is absent and a medicine was present in various strength in the market (Table 4), it might lead to wrong dose taken by patient and sometimes might cause overdose of medicine.

This study also identified those 6 prescriptions out of 200 containing two medicine of the same type (Table 3).

This frequency was low but may cause serious problem because in most of the cases they caused serious drug-drug interaction and sometimes may lead to overdose. A total of 366 drug interactions were also identified from 200 prescriptions in this study. Among these interactions 46 (12.57\%) were serious, 195 (53.28\%) were significant and $125(34.15 \%)$ were minor drug interaction (Figure 3).

Identification of prescription errors also showed that about $15 \%$ prescriptions orders were for the patients with kidney and urinary problems and there were no dose adjustment. Missing of date of admission in the prescriptions were $4.5 \%$. The number of prescriptions containing two or more than two medicines used for the same purpose was $8.5 \%$.

Different studies have proposed different strategies to prevent medication errors. These include optimizing the medication process, eliminating situational risk factors, oversight and error interception and interdisciplinary teams (Williams 2007; Moyen et al., 2009). It was demonstrated that the healthcare system without pharmacists is unable to effectively cope up with most medicine related issues.

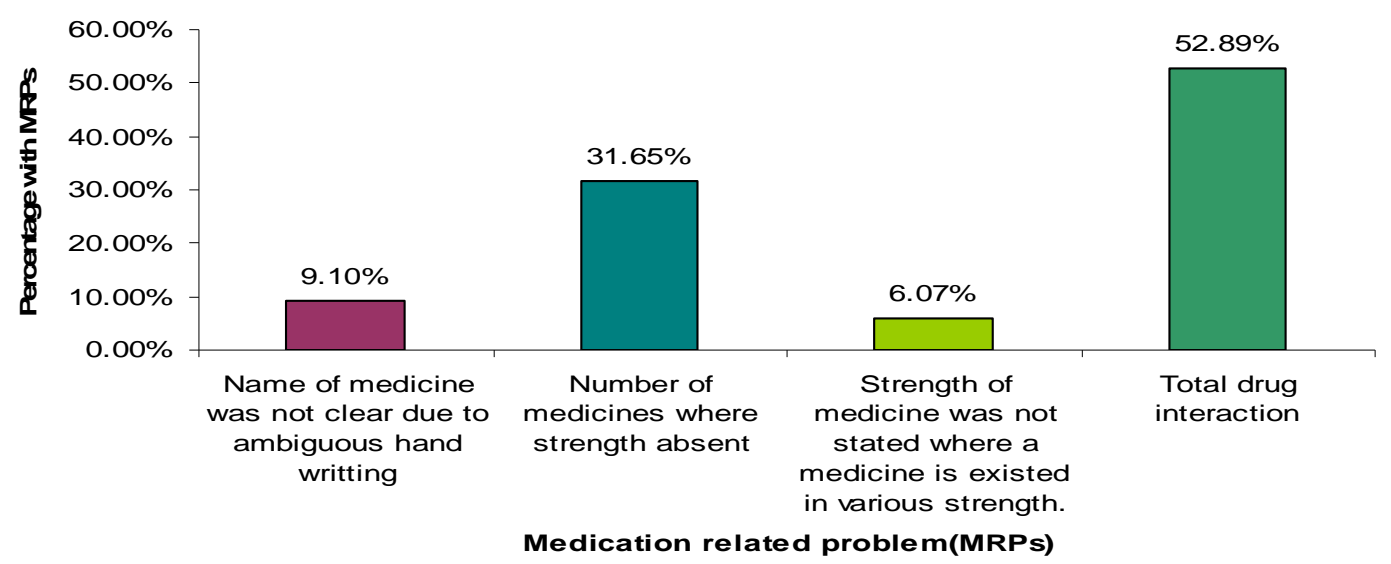

Figure 2. MRPs identified from the prescription

Table 2. Strength of medicine was not stated where a medicine is existed in various strength:

\begin{tabular}{ll}
\hline Drugs & Strength present in the market \\
\hline 1. Diclofenac suppository & $12.5 \mathrm{mg}, 50 \mathrm{mg}$ \\
2. Inj Ceftriaxone & $1 \mathrm{gm} / \mathrm{vial}, 250 \mathrm{mg}, 500 \mathrm{mg}$ and $2 \mathrm{gm} / \mathrm{vial}$. \\
3. Paracetamol Suppository & $125 \mathrm{mg}, 250 \mathrm{mg}$ and $500 \mathrm{mg}$ \\
4. Inj Ceftriaxone & $1 \mathrm{gm}, 250 \mathrm{mg}, 500 \mathrm{mg}$ \\
5. Inj. Amoxicillin & $250 \mathrm{mg}, 500 \mathrm{mg} / \mathrm{vial}$ \\
\hline
\end{tabular}


Table 3. Prescribing two drugs of the same type.

\begin{tabular}{lll}
\hline \multicolumn{2}{l}{ Drugs } & Class and purpose \\
\hline I. $\quad$ Tramadol and Nalbuphine & $\begin{array}{l}\text { Both are opoid analgesics and used for analgesics and } \\
\text { antipyretic reason }\end{array}$ \\
II. $\quad$ Paracetamol and Ketorolac & $\begin{array}{l}\text { Both are non-opoid analgesics and used for analgesics and } \\
\text { antipyretic reason without adjusting the dose }\end{array}$ \\
I. Naproxen and Aceclofenac & $\begin{array}{l}\text { Both are NSAIDs and used for inflammation and rheumatic } \\
\text { disease }\end{array}$ \\
V. $\quad$ Omeprazole and Rabeprazole & Both are Proton pump inhibitor and used for peptic ulcer \\
\hline
\end{tabular}

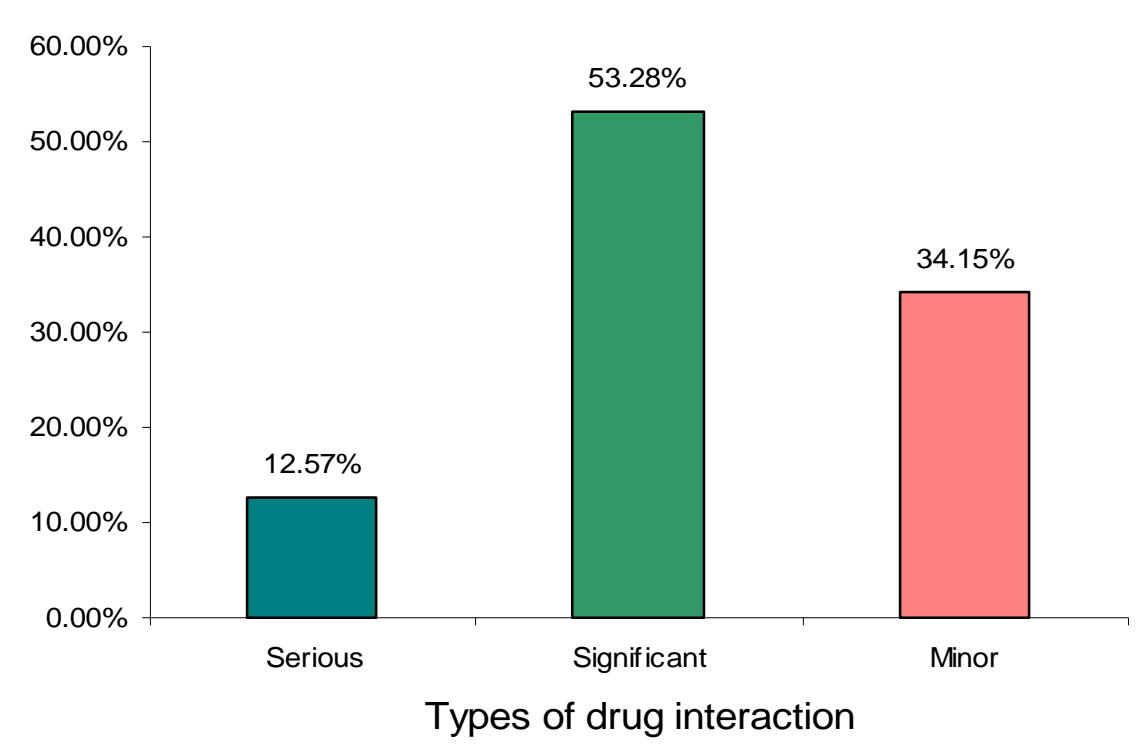

Figure 3. Various types of drug interaction present in the prescriptions.

Many studies have emphasized inclusion of the clinical pharmacist in the health care team to help identify MEs, contribute to the rationalization of drug therapy, and potentially prevent negative consequences, leading to increased medication safety (Williams 2007; Moyen et al., 2009; Went et al., 2010; Vessal 2010).

Finally, it must also be noted that this study didn't explore the severity of errors, outcome of treatment or reasons for errors. The use of guidelines rather than clinical opinions to determine error and the small number of patients included in the study must also be noted.

\section{Conclusion}

In conclusion, medication errors in the prescribing phase were highly prevalent. The errors reported in this study clearly show that there are multiple causes for prescribing errors. The lack of close supervision for the prescribing medical interns, along with the absence of the clinical pharmacist could have made things worse.
Hospital managers should strive to create better awareness about the possibility of medication errors at the prescribing phase among health care professionals. Introduction of routine checks with close supervision of the prescribing intern physicians are strongly recommended. We also recommend the inclusion of clinical pharmacists in the health care team of the hospitals.

\section{Acknowledgement}

The authors are thankful to Rafatullah and TMSS Community Hospital, Bagora, Bangladesh for their cooperation and support to carry out this survey based research.

\section{References}

Al-Dhawailie, A.A. 2011. Inpatient prescribing errors and pharmacist intervention at a teaching hospital in Saudi Arabia. Saudi Pharm J. 19, 193-196. 
Barker, K.N., Flynn, E.A., Pepper, G.A., Bates, D.W. and Mikeal, R.L. 2002. Medication errors observed in 36 health care facilities. Arch Intern Med.162, 1897-1903.

Bates, D.W., Cullen, D.J., Laird, N. and Petersen, L. 1995. Incidence of adverse drug events and potential adverse drug events. Implications for prevention. J. Am. Med. Assoc. 274, 29-34.

Blum, K.V., Abel, S.R. and Urbanski, C.J. 1988. Medication error prevention by pharmacists. Am. J. Hosp. Pharm. 45, 1902-1903.

Bobb, A., Gleason, K., Husch, M., Feinglass, J., Yarnold, P.R. and Noskin, G.A. 2004. The epidemiology of prescribing errors: the potential impact of computerized prescriber order entry. Arch Intern Med. 164, 785-792.

Castelino, R.L., Sathvik, B.S., Parthasarathi, G., Gurudev, K.C., Shetty, M.S. and Narahari, M.G. 2011. Prevalance of medocation-related problems among patients with renal compromise in an Indian hospital. J. Clin. Pharm. Therapeutics 36, 481-487.

Dean, B., Schachter, M., Vineem, C. and Barber. N. 2000. Prescribing errors in hospital inpatients, their incidence and clinical significance. Quality Saf. Health Care 77, 340-344.

Dean, B., Schachter, M., Vineem, C. and Barber. N. 2000. Causes of prescribing errors in hospital inpatients, a prospective study. Lancet 359, 1373-1378.

Dean, B., Barber, N. and Schachter, M .2000. What is a prescribing error? Quality Health Care 9, 232-237.

Dean, B., Schachter, M., Vincent, C. and Barber, N. 2002. Prescribing errors in hospital inpatients: their incidence and clinical significance. Quality Saf. Health Care. 11, 340-344.

Fahimi, F., Sistanizadb, M., Abrishami, R. and Baniasadi, S. 2007. An observational study of errors related to the preparation and administration of medications given by infusion devices in a teaching hospital. Int. J. Pharm. Res. 6, 295-299.

Folli, H.L., Poole, R.L. and Benitz, W.E. 1987. Medication error prevention by clinical pharmacists in two children's hospitals. Pediatrics 79, 718-22.

Hawkey, C.J., Hodgson, S., Norman, A., Danseshmend, T.K. and Garner, S.T. 1990. Effect of reactive pharmacy intervention on quality of hospital prescribing. British Med. J. 300, 986990.
Kohn, L.T., Corrigan, J.M. and Donaldson, M.S. 1999. To err is human. Building a safer health System. Washington. DC: National Academy Press.

Kozer, E., Scolnik, D., Macpherson, A., Rauchwerger, D. and Koren, G. 2005. Using a preprinted order sheet to reduce prescription errors in a pediatric emergency department: a randomized, controlled trial. Pediatrics 116, 1299-1302.

Kozer, E., Scolnik, D., Macpherson, A., Rauchwerger, D. and Koren, G. 2006. The effect of a short tutorial on the incidence of prescribingerrors in pediatric emergency care, Can. J. Clin. Pharmacol. 13, 285-291.

Lesar, T.S., Lomaestro, B.M. and Pohl, H. 1997. Medicationprescribing errorsin a teaching hospital. A 9-year experience. Arch Intern. Med. 157, 1569-1576.

Lewis, P.J., Dornan, T., Taylor, D., Tully, M.P., Wass, V. and Ashcroft, D.M. 2009. Prevalence, incidence and nature of prescribing errors in hospital inpatients a systematic review, Drud. Saf. 32, 379-389.

Mandal, K. and Fraser, S.G. 2005. The incidence of prescribing errors in an eye hospital, BMC Ophthalmology 5, 4-10.

Moyen, E., Camire, E. and Stelfox, H.T. 2008. Medication errors in critical care. Crit Care 12, 208.

Moyen, E., Camire, E. and Stelfox, H.T. 2009.Medication errors in critical care: risk factors, prevention and disclosure. CMAJ 180, 936-943.

Reason, J.T. 1990. Human Error. Cambridge: Cambridge University Press.

Rinke, M.L., Moon, M., Clark, J.S., Mudd, S. and Miller, M.R. 2008. Prescribing errors in a pediatric emergency department, Pediator. Emerg. Care 24, 1-8.

Smith, J. 1999. Study into medical errors planned for the UK. British Med. J. 319, 1091.

Vessal, G. 2010. Detection of prescription errors by a unit based clinical pharmacist in a nephrology ward. Pharm. World Sci. 32, 59-65.

Went K, Antoniewicz P, Corner DA, et al. 2010. Reducing prescribing errors: Can a well designed electronic system help? J. Eval. Clin. Pract. 16, 556-559.

Williams, D.J.P. 2007. Medication errors. J. R. Coll. Physicians, Edin. 37, 343-346. 